\title{
Is beryllium ultra-depletion in solar-type stars linked to the presence of a white dwarf companion?
}

\author{
S. Desidera ${ }^{1}$, V. D’Orazi ${ }^{1,2,3}$, and M. Lugaro ${ }^{4,3}$ \\ 1 INAF Osservatorio Astornomico di Padova, vicolo dell'Osservatorio 5, 35122 Padova, Italy \\ e-mail: silvano.desidera@oapd.inaf.it \\ 2 Department of Physics and Astronomy, Macquarie University, Balaclava Rd, NSW 2109, Australia \\ 3 Monash Centre for Astrophysics, School of Physics and Astronomy, Monash University, VIC 3800, Australia \\ ${ }^{4}$ Konkoly Observatory, Hungarian Academy of Sciences, PO Box 67, 1525 Budapest, Hungary
}

Received 21 September 2015 / Accepted 16 October 2015

\section{ABSTRACT}

\begin{abstract}
Context. Abundance studies of solar-type stars revealed a small fraction of objects with extreme depletion of beryllium. Aims. We investigate the possible link between the beryllium depletion and the presence of companions.

Methods. The classical methods (radial velocity, astrometry, imaging) used to search for binary companions were exploited. We also performed a chemical analysis to identify binaries by the alteration in abundances that is produced by the accretion of material lost by a former evolved companion.

Results. We found that all the four previously investigated stars that were found to be ultra-depleted in Be are binaries. In two cases the companion is a white dwarf, and in the other two cases the companion might be a white dwarf or a main-sequence star. One new barium star was identified.

Conclusions. We speculate that the interaction with the white dwarf progenitor caused an alteration in the abundance pattern of the star, which resulted in severe beryllium depletion. Possible mechanisms such as thermohaline mixing, episodic accretion, and rotational mixing are discussed. We also briefly discuss predictions for validating this scenario.
\end{abstract}

Key words. stars: abundances - stars: AGB and post-AGB - binaries: general - stars: late-type - white dwarfs

\section{Introduction}

The determination of light-element abundances ( $\mathrm{Li}, \mathrm{Be}$, and $\mathrm{B}$ ) provides fundamental information on stellar interiors. Thanks to their fragile nature, these three elements can be used to probe the mixing processes beneath the stellar surface, where they can be destroyed primarily by proton-capture reactions. Temperatures at which these phenomena occur are approximately 2.5 million $\mathrm{K}$ for $\mathrm{Li}$ and 4 million $\mathrm{K}$ for $\mathrm{Be}$ and $\mathrm{B}$. The spectral lines of B reside only in the far UV, which prevents gathering B abundances without going above the atmosphere. On the other hand, lithium is remarkably easier to observe (its resonance doublet occurs in the red portion of the spectrum at $6708 \AA$ ), but because there are multiple sites for the creation and destruction of $\mathrm{Li}$, the interpretation of $\mathrm{Li}$ abundances is sometimes complicated (see e.g. Karakas \& Lattanzio 2014, and references therein). Beryllium provides a clearer picture because it has only one mechanism of production: spallation reactions in the vicinity of supernovae or in the ambient interstellar gas. The resonance lines of Be II are found at 3130.421 and $3131.065 \AA$, which are still observable with ground-based telescopes. For this reason, a plethora of observational projects have been commenced in the past decades to ascertain the Be content in different types of stars and stellar populations (old, metal-poor stars, globular clusters, solar-type stars and open clusters, including stars with exo-planets; see e.g. Boesgaard \& King 2002; Boesgaard et al. 2004; Santos et al. 2004; Pasquini et al. 2007; Smiljanic et al. 2009, 2010, 2011).

Recently, Takeda et al. (2011) presented Be abundances for a sample of 118 solar analogues (along with 87 FGK stars) and found that, although most of the Sun-like stars share a Be content similar to the Sun (roughly \pm 0.2 dex within the solar value), four stars exhibit significant Be depletion by more than a factor of 100. They noted that these stars belong to the group with the slowest rotational velocities, where differential rotation between the core and the envelope may be higher and promote enhanced mixing leading to Be depletion (Bouvier 2008). However, it is not straightforward to explain why this process should only occur in a tiny fraction of the relatively slowly rotating Sun-like stars and not not in the large majority of these objects, or in other words, why it is not ubiquitous.

One of the four $\mathrm{G}$ dwarfs with very high beryllium depletion identified by Takeda et al. (2011), HIP 64150, was recently shown to have a white dwarf (WD) companion at a projected separation of about 18 AU (Crepp et al. 2013). Motivated by this discovery, we examine the multiplicity of the four Bedepleted $\mathrm{G}$ dwarfs to determine whether binarity is a unique case for HIP 64150 or a common feature of these stars. We base our analysis on the standard techniques used to identify stellar companions (e.g. radial velocity variability) and, more specifically for WD companions, on indirect evidence of mass transfer from a former AGB star, seen as anomalies in their chemical abundances.

\section{Binarity}

\section{1. $H I P 64150=H D 114174$}

As mentioned above, HIP 64150 has a WD companion discovered as part of the TRENDS project (AO follow-up of stars with long-period RV trends) by Crepp et al. (2013). The WD 
companion lies at a projected separation of about $18 \mathrm{AU}$ and the dynamical lower limit to its mass is $0.26 M_{\odot}$. Crepp et al. (2013) derived from their $J H K$ photometry an effective temperature around $8000 \mathrm{~K}$ and cooling age of about $3 \mathrm{Gyr}$ for the WD. Matthews et al. (2014) estimated a significantly lower effective temperature and longer cooling age including their $L^{\prime}$ and $M$-band photometry. Regardeless of these discrepancies, the long cooling age is compatible with the slow stellar rotation and low level of activity of the unevolved companion star.

\subsection{HIP $17336=$ HD 23052}

HIP 17336 is a spectroscopic and astrometric binary (Scarfe \& Griffin 2012, and HIPPARCOS orbital solution). The orbit has a period of $2.8 \mathrm{yr}$ and an eccentricity of 0.125 . The detection of the astrometric signature of the binary orbit from HIPPARCOS data allowed Scarfe \& Griffin (2012) to solve the inclination ambiguity from RV and infer a true companion mass of between $0.56 \mathrm{e}$ $0.62 M_{\odot}$, compatible with a late-K or early-M dwarf or a WD. In the latter case, considering its current separation, the system should have been evolved through the common-envelope phase. If the companion is a main-sequence star, the expected magnitude difference is about 3.8 in $\mathrm{V}$ and 1.8 in $K_{\mathrm{s}}$. Therefore, the case of a main sequence companion can be easily confirmed or ruled out with near-infrared observations (see e.g. Mazeh et al. 2002). Galex far- and near-UV (FUV and NUV) and fluxes do not provide evidence for the presence of a hot WD.

\section{3. $H I P 32673=H D 49178$}

This star is reported to have astrometric acceleration from HIPPARCOS. Nordström et al. (2004) reported a possible variability (probability of constant RV 5\% from two-epoch observations). To further evaluate the binarity of the object, we retrieved 12 RV measurements from the ELODIE ${ }^{1}$ and SOPHIE $^{2}$ archive. The four ELODIE spectra were taken in 2004-2005 and the eight SOPHIE spectra in late 2006. While the time information is partially masked in the SOPHIE data archive, preventing an accurate orbit determination, an RV regular trend of $1.6 \mathrm{~km} \mathrm{~s}^{-1}$ over the baseline of the observations, ELODIE data also exhibit highly significant variations, and when the Nordström et al. (2004) RV is taken into account, the peak-to-valley variations are of $15 \mathrm{~km} \mathrm{~s}^{-1}$. A tentative periodicity of the order of $2.5-3 \mathrm{yr}$ is deduced from ELODIE and SOPHIE RVs. The cross-correlation function (CCF) profiles from ELODIE and SOPHIE indicate a single-line SB. GALEX FUV and NUV fluxes do not provide evidence for the presence of a hot WD.

\section{4. $H I P 75676=H D 138004$}

This star shows astrometric acceleration (seven-parameter solution) in HIPPARCos. Two-epoch RV observations from Nordström et al. (2004) show that this is an RV variable (rms RV $1.9 \mathrm{~km} \mathrm{~s}^{-1}$, baseline 354d). Furthemore, Riddle et al. (2015) reported that the star is a spectroscopic binary according to private communication by D. Latham. Therefore, this star probably also has a companion with a period of a few years. From the signature of pollution by an AGB star that we found in Sect. 3, we conclude that this companion must be a white dwarf. No additional companions were detected in the AO survey by Metchev \& Hillenbrand (2009), however, there appears to also be a wide

\footnotetext{
1 http://atlas.obs-hp. fr/elodie

2 http://atlas.obs-hp.fr/sophie/
}

third component at 40 arcsec projected separation, the $\mathrm{K}$ dwarf $\mathrm{BD}+432500 \mathrm{~B}$. This latter was recently resolved into a 0.4 arcsec close visual binary by Riddle et al. (2015).

\section{Abundance analysis}

Along with searching for the dynamical signature from a companion and the direct search through imaging observations, an alternative way to infer the presence of a WD companion is to search for abundance anomalies that are linked to the accretion of material produced during the AGB phase, enriched in $\mathrm{C}$ and elements produced by the slow neutron-capture process (the $s$ process, Busso et al. 1999). Barium stars represent the classical example of this process (Jorissen et al. 1998).

\subsection{Data and analysis procedure}

To derive the chemical abundances, we exploited spectra from the High Resolution Echelle Spectrograph (HIRES, Vogt et al. 1994) that are available from the Keck archive ${ }^{3}$ for stars HIP 64150 and HIP 75676. HIP 64150 was observed on June 9, 1997 under program N10H (PI: J. Lissauer) employing the 0.6 arcsec slit, which results in a spectral resolving power of about $R \sim 55000$. The wavelength coverage ranges from 3750 to $6200 \AA$. The signal-to-noise ratio per pixel (S/N) is about 200 at $\sim 5850 \AA$. For HIP 75676 the spectrum was acquired during the observing program N03H (PI: J. Butler) on June 16, 2003; a 0.9 arcsec slit was adopted, which implies a resolution of $R \sim 45000$ (spectral coverage 3750-6200 $⿱$ ). The S/N around the Ba II line at $5853 \AA$ is 300 . For the other two sample stars, HIP 32673 and HIP 17336, we used ELODIE spectra available from the archive ${ }^{4}$. The spectrograph, which was mounted at the Cassegrain focus of the $1.93 \mathrm{~m}$ telescope at the Observatoire de Haute-Provence (CNRS), covers a $3000 \AA$ range (3850$6800 \AA$ ) with a spectral resolution of $R=42000$. Observations were carried out on February 17, 2004 (PI: T. Mazeh) and on February 18, 2005 (PI: T. Mazeh) for HIP 32673 and HIP 17336, respectively. The $\mathrm{S} / \mathrm{N}$ are around 70 and 50 at $5850 \AA$. In Fig. 1 we show a portion of the spectra for the four sample stars in the wavelength regions 4180-4220 $\AA$ (upper panel) and 5830-5870 A (lower panel).

Abundance analysis was carried out via spectral synthesis with MOOG (Sneden 1973, 2011 version) and the Kurucz grid of model atmospheres with no overshooting (Castelli \& Kurucz 2003). For $s$-process element abundances, we adopted the same line lists as in D'Orazi et al. (2012), to which we refer for details on atomic oscillator strengths, hyperfine structure information, isotopic shifts, and adopted ratios, and derived abundances for the Sun and Arcturus. Here we briefly recall that a singleline treatment was assumed for the Y II and Zr II lines (4398 $\AA$ and $4209 \AA$, respectively), while hyperfine structure and isotopic shifts were included for the Ba II feature at $5853 \AA$ and La II at $4087 \AA$. An example of the spectral synthesis procedure is shown in Fig. 2 for star HIP 64150.

Carbon abundances were obtained by exploiting the $\mathrm{CH}$ molecular band at $4300 \AA$. The line list comes from Plez (priv. comm.). For consistency with our previous analysis and $s$-process element abundances, we assumed $T_{\text {eff, } \odot}=$ $5770 \mathrm{~K}, \log g_{\odot}=4.44 \mathrm{~cm} \mathrm{~s}^{-2}, \xi_{\odot}=1.1 \mathrm{~km} \mathrm{~s}^{-1}$, and

\footnotetext{
3 http://www2 . keck.hawaii.edu/koa/public/koa.php

4 http://atlas.obs-hp.fr/elodie/
} 
S. Desidera et al.: Is beryllium ultra-depletion in solar-type stars linked to the presence of a white dwarf companion?

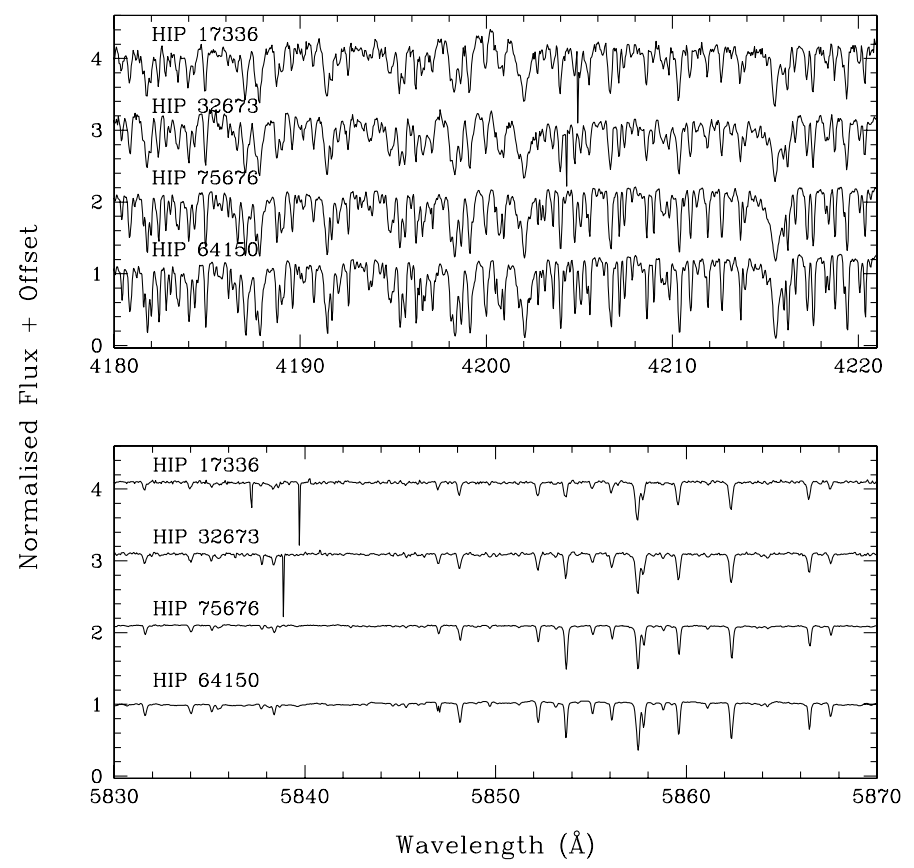

Fig. 1. Example of the spectra for our sample stars in two different wavelength regions.

$\log n(\mathrm{Fe})_{\odot}=7.52$. Our analysis results in a carbon solar abundance of $A(\mathrm{C})_{\odot}=8.30$, which is lower than typical literature values, such as $A(\mathrm{C})=8.52 \pm 0.06$ by Grevesse \& Sauval (1998). However, since our analysis is strictly differential with respect to the Sun, this does not affect our conclusions.

Stellar parameters $\left(T_{\mathrm{eff}}, \log g\right.$, microturbulence velocity $\left.\xi\right)$ and metallicity $([\mathrm{Fe} / \mathrm{H}])$ were retrieved from the study by Takeda et al. (2007), who published $\mathrm{Li}$ abundances for our four stars (within a total sample of 118 stars). We refer to Takeda et al. (2007) for an extensive discussion on the accuracy of the atmospheric parameters derived trough the spectroscopic procedure (excitation/ionisation equilibrium condition and curve-ofgrowth matching). Uncertainties on the derived abundances were evaluated following the same approach as in D'Orazi et al. $(2009,2011,2012)$. The total internal errors related to the bestfit determination and to the adopted set of stellar parameters range from 0.13 to $0.15 \mathrm{dex}$, depending on the species, with $\mathrm{Ba}$ affected by larger uncertainties given the almost saturated behaviour of the line at $5853 \AA$ ( $\xi$ is by far the dominant source of error). Following Takeda et al. (2007), we adopted errors of $20 \mathrm{~K}, 0.05 \operatorname{dex}, 0.10 \mathrm{~km} \mathrm{~s}^{-1}$, and 0.02 dex for $T_{\text {eff }}, \log g$, $\xi$, and input metallicity $[\mathrm{Fe} / \mathrm{H}]$, respectively.

\subsection{Results}

Our results are reported in Table 1, where we list stellar parameters (from Takeda et al. 2007), $V$ magnitudes and $B-V$ colours, along with abundances for carbon and $s$-process elements. We found a solar composition in terms of $\mathrm{Y}, \mathrm{Zr}, \mathrm{Ba}$, and La for HIP 32673, with a mean value $[s / \mathrm{Fe}]=0.01 \pm 0.01$ $(\mathrm{rms}=0.03)$. The star HIP 17336 exhibits, on the other hand, a sub-solar $s$-process element content, being the average $[s / \mathrm{Fe}]=$ $-0.27 \pm 0.03$; in this case we could not derive the La abundance because of a cosmic ray falling on the La line at $4087 \AA$.

Interestingly, we identified a new Ba star, HIP 75676: this star is characterised by a high over-abundance of $s$-process

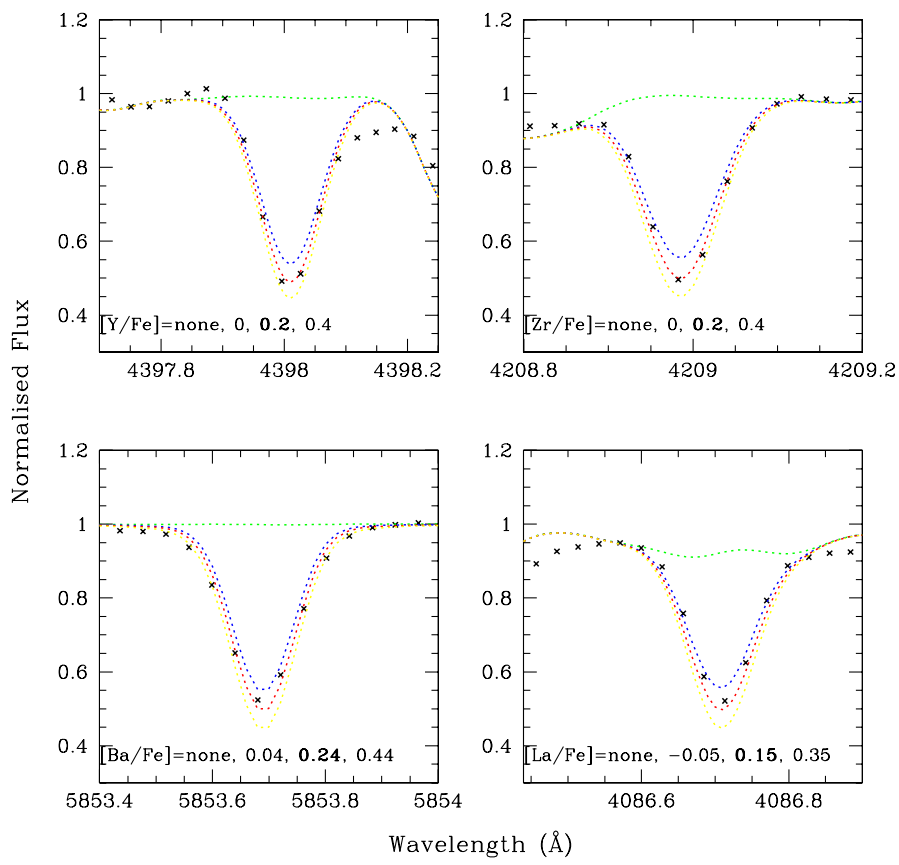

Fig. 2. Spectral synthesis for star HIP 64150 for the Y II, Zr II, Ba II, and La II lines.

elements, both first-peak $\mathrm{Y}$ and $\mathrm{Zr}$ and second-peak Ba and $\mathrm{La}$, with a mean value of $[s / \mathrm{Fe}]=1.16 \pm 0.02$.

Finally, HIP 64150 reflects a mild super-solar abundance pattern in terms of $s$-process elements $([s / \mathrm{Fe}]=0.21 \pm 0.01)$. This star has been previously analysed by Ramírez et al. (2009), who derived $[\mathrm{Ba} / \mathrm{Fe}]=0.15 \pm 0.05$; the small difference $(\Delta=$ $0.09 \pm 0.10)$, which is within the measurement uncertainties, can be explained by the difference in the microturbulence velocity since we have adopted the value of $\xi=1.03$ by Takeda et al. (2007), while Ramírez et al. (2009) obtained $\xi=1.14 \mathrm{~km} \mathrm{~s}^{-1}$.

\section{Discussion}

Our investigation of the multiplicity of the four ultra Be-depleted $\mathrm{G}$ dwarfs identified by Takeda et al. (2011) shows that all these objects are binaries, with companions with orbital period from a few years to several tens of years. At least two of them have WD companions: one was shown by direct detection, the other was deduced based on its $s$-process overabundance. The remaining two cases are also compatible with a WD companion, especially HIP 17336, for which the dynamical mass of the companion is between 0.56 to $0.62 M_{\odot}$.

We investigated the multiplicity of the remaining 114 solar analogues in the Takeda et al. (2011) sample in the SB9 (Pourbaix et al. 2004), HIPPARCOS (Perryman et al. 1997), and WDS catalogues (Mason et al. 2001). We found that several stars with normal Be abundance have stellar companions with a variety of separations, encompassing the range of ultra Be-depleted stars (Table 2). None of these companions, however, are WDs. This indicates that even though a WD companion needs to be confirmed for two of our targets, the observed Be depletion must be linked not only to the presence of a stellar companion, but specifically to the case when the companion star already evolved throughout the red giant and AGB phases into a WD. The presence of a WD companion within a few AU implies some interaction between the G dwarf and the WD progenitor during its red giant or AGB phase through a common-envelope phase 
Table 1. Stellar parameters, $V$ magnitudes, $B V$ colours, $C$ and $s$-process element abundances.

\begin{tabular}{|c|c|c|c|c|c|c|c|c|c|c|c|c|}
\hline Star & Alt. name & V & $\begin{array}{c}B-V \\
5\end{array}$ & $\begin{array}{l}T_{\text {eff }} \\
(\mathrm{K})\end{array}$ & $\begin{array}{c}\log g \\
\left(\mathrm{~cm} \mathrm{~s}^{-2}\right)\end{array}$ & $\begin{array}{c}{[\mathrm{Fe} / \mathrm{H}]} \\
(\mathrm{dex})\end{array}$ & $\begin{array}{c}\xi \\
\left(\mathrm{km} \mathrm{s}^{-1}\right)\end{array}$ & $\begin{array}{c}{[\mathrm{C} / \mathrm{Fe}]} \\
(\mathrm{dex})\end{array}$ & $\begin{array}{c}{[\mathrm{Y} / \mathrm{Fe}]} \\
(\mathrm{dex})\end{array}$ & $\begin{array}{c}{[\mathrm{Zr} / \mathrm{Fe}]} \\
(\mathrm{dex})\end{array}$ & $\begin{array}{c}{[\mathrm{Ba} / \mathrm{Fe}]} \\
(\mathrm{dex})\end{array}$ & $\begin{array}{r}{[\mathrm{La} / \mathrm{Fe}]} \\
(\mathrm{dex})\end{array}$ \\
\hline HIP 64150 & HD 114174 & 6.78 & 0.667 & 5747 & 4.45 & 0.07 & 1.03 & 0.00 & 0.20 & 0.20 & 0.24 & 0.18 \\
\hline HIP 75676 & HD 138004 & 7.48 & 0.676 & 5755 & 4.40 & -0.09 & 0.92 & 0.21 & 1.20 & 1.10 & 1.20 & 1.15 \\
\hline HIP 32673 & HD 49178 & 8.07 & 0.677 & 5724 & 4.57 & 0.06 & 0.95 & -0.05 & 0.00 & 0.00 & 0.05 & -0.02 \\
\hline HIP 17336 & HD 23052 & 7.07 & 0.659 & 5671 & 4.54 & -0.13 & 0.94 & -0.12 & -0.30 & -0.20 & -0.30 & $\ldots$ \\
\hline
\end{tabular}

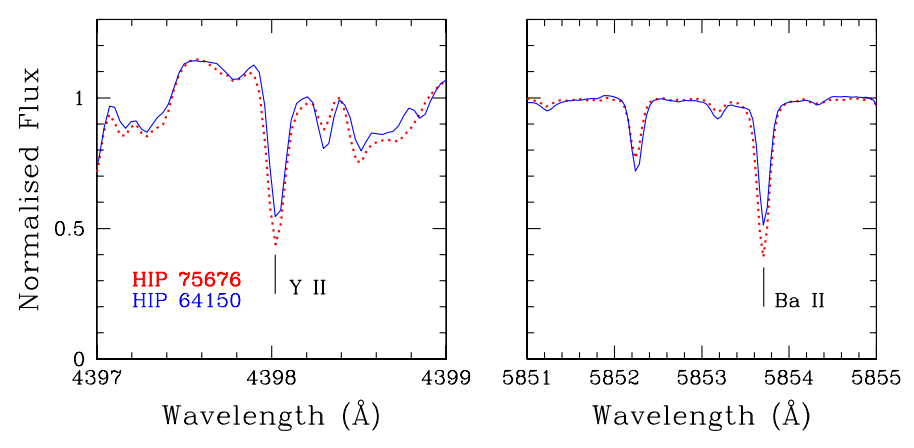

Fig. 3. Comparison of HIP 64150 (blue solid line) and HIP 75676 (red dotted line)

and/or wind accretion, depending on the initial binary configuration. Accretion of mass from an AGB companion is the standard explanation for the $\mathrm{C}$ - and s-process-enhanced composition of Ba stars, as in the case of the Ba star found in our sample, and it is possible also for the mildly enriched star reported here. The C- and s-process enrichment is expected only in AGB stars of masses roughly above $1.5 M_{\odot}$ because they experience the third dredge-up (Iben \& Renzini 1983). The other two stars that show no $s$-process enhancements could also have experienced the same binary evolution path leading to Be depletion as the stars showing s-process enhancements, but with interaction and accretion from an AGB companion of initial mass lower than $1.5 M_{\odot}$.

It is expected that the Be abundance in the accreted material is close to zero, since the envelope of AGB stars reaches very deep in mass to hot layers and strong Be depletions are observed already in the previous red giant phase (Takeda \& Tajitsu 2014). However, accretion from an AGB star is not enough by itself to quantitatively explain the observed level of depletion. The mass of the convective surface layer of these solar analogues is of the order of $0.02 M_{\odot}$. It would be necessary to accrete $2 M_{\odot}$ of material from an AGB star to achieve the ratio of initial to accreted material of less than 1/100 required to explain the Be depletions of two orders of magnitude. This is clearly not feasible. A possibility is that the material that we see at the stellar surface represents pure AGB material. Evidence of the presence of pure accreted material may come from a systematic depletion in $\mathrm{C}$ in the two stars that do not show s-process enhancement. The companions would have experienced the first dredge-up during the red giant phase, which reduces $\mathrm{C}$, but not the third dredge-up during the AGB, which increases $\mathrm{C}$. However, the $\mathrm{C}$ depletion during the first dredge-up depends on the initial stellar mass and may be too small to be detected $(<0.1$ dex for an initial mass of the WD $<1.2 M_{\odot}$ ). Furthermore, it is difficult to find a physical reason why the accreted material should not mix into the convective surface layer of these stars. Another three possible mechanisms to explain the origin of extreme Be depletion in solar-type stars are thermohaline mixing, episodic accretion, and rotational mixing.

\subsection{Thermohaline mixing}

Accretion from a red giant or AGB companion produces a change in the mean molecular weight of the external layers of the star, since the accreted material is more rich in He than the material on the secondary. The change in mean molecular weight is of the order of less than one to a few percent, depending on the amount of material accreted and if the companion experienced only the first dredge-up or also the third dredge-up. In any case, it would favour the development of mixing processes such as thermohaline mixing, as investigated in the context of carbonenhanced metal-poor (CEMP) stars, together with effect of gravitational settling (e.g. Stancliffe \& Glebbeek 2008). Depending on the amount of mass accreted and the initial mass of the companion, it is qualitatively expected that more efficient thermohaline mixing has been at work to deplete Be in the stars considered here than in similar stars that did not accrete from an evolved companion, but only a detailed model can confirm this quantitatively.

\subsection{Episodic accretion}

Baraffe \& Chabrier (2010) and Viallet \& Baraffe (2012) showed that bursts of episodic accretion phenomena during the pre-MS phase have significant effects on the stellar structure that imply severe destruction of $\mathrm{Li}$ and $\mathrm{Be}$. The accretion bursts considered in these studies have rates of the order of $10^{-4}-10^{-3} M_{\odot} / \mathrm{yr}$, which may occur only in rather extreme conditions of protostellar clouds. The mass-loss rate of AGB stars is between $10^{-7}$ and $10^{-4} M_{\odot} / \mathrm{yr}$, and these high required accretion values are also well beyond our expectation for the wind-accretion scenario, even if we assumed that significantly higher mass-loss rates may be achieved on short timescales. Conversely, common-envelope evolution may be rather fast, although much more uncertain, allowing in principle high accretion rates. It should also be taken into account that the accretors considered in Baraffe \& Chabrier (2010) and Viallet \& Baraffe (2012) are protostars at early evolutionary phases, very different from the mature solar-type stars onto which the accretion would occur at the end of the AGB phase of the WD companion progenitor. In these cases lower accretion rates might be enough to trigger changes in the internal structure capable of destroying Be.

\subsection{Rotational mixing}

The accretion of material lost by the WD progenitor also implies accretion of angular momentum on the companion, altering the rotational evolution of the companion (Jeffries \& Stevens 1996). Several objects with WD companions showing excesses of rotation and activity for their ages have been 
Table 2. Binaries in the Takeda et al. (2011) sample and their characteristics.

\begin{tabular}{|c|c|c|c|c|c|c|c|c|c|c|}
\hline Star & Alt. name & $\mathrm{A}(\mathrm{Be})$ & Period & $\begin{array}{c}\text { Sep } \\
\text { arscec }\end{array}$ & $\begin{array}{c}a \\
\mathrm{AU} \\
\end{array}$ & $e$ & $\begin{array}{l}\text { M1 } \\
M_{\odot} \\
\end{array}$ & $\begin{array}{l}\text { M2 } \\
M_{\odot} \\
\end{array}$ & Type & Reference \\
\hline HIP 64150 & HD 114174 & $<-0.88$ & - & 0.68 & $18.0^{*}$ & - & 1.05 & 0.54 & $\mathrm{SB}+\mathrm{VB}$ & Cr13, Ma14 \\
\hline HIP 75676 & HD 138004 & $<-0.96$ & - & - & - & - & 0.96 & - & $\mathrm{SB}+\mathrm{AB}+\mathrm{Ba}$ & this paper \\
\hline HIP 32673 & HD 49178 & $<-0.78$ & $\sim 1000 \mathrm{~d}$ & - & - & - & 1.00 & - & $\mathrm{SB}+\mathrm{AA}$ & this paper \\
\hline HIP 17336 & HD 23052 & $<-0.85$ & $1030 \mathrm{~d}$ & - & - & 0.125 & 0.95 & 0.59 & $\mathrm{SB}+\mathrm{AB}$ & $\mathrm{Sc} 12$ \\
\hline HIP 7244 & HD 9472 & 1.23 & - & 2.8 & $97.0^{*}$ & - & 0.98 & 0.11 & VB & WDS \\
\hline HIP 7918 & HD 10307 & 1.25 & $7122 d$ & 0.7 & 8.9 & 0.10 & 1.08 & 0.43 & $\mathrm{SB}+\mathrm{AA}+\mathrm{VB}$ & SB9, HIP, WDS \\
\hline HIP 8486 & HD 11131 & 1.33 & $2471 d$ & - & 4.0 & 0.45 & 0.97 & 0.42 & $\mathrm{AB}$ & Go07 \\
\hline HIP 19911 & HD 26990 & 1.33 & $5.68 \mathrm{yr}$ & 0.10 & 3.10 & 0.81 & 0.95 & 0.85 & $\mathrm{SB}, \mathrm{AA}, \mathrm{VB}$ & HIP, To14, To15 \\
\hline HIP 20441 & HD 27685 & 1.16 & $4623 d$ & - & 6.16 & - & 1.01 & 0.45 & $\mathrm{SB}+\mathrm{AA}$ & HIP, To14 \\
\hline HIP 20741 & HD 28099 & 1.25 & - & 0.43 & $19.8^{*}$ & - & 1.08 & 0.16 & $\mathrm{SB}+\mathrm{VB}$ & Gu05 \\
\hline HIP 25414 & HD 35073 & 1.25 & - & - & - & - & 1.02 & - & AA & HIP \\
\hline HIP 40118 & HD 68017 & 1.08 & - & 0.6 & 13.0 & - & 0.81 & 0.15 & $\mathrm{SB}+\mathrm{VB}$ & $\mathrm{Cr} 12$ \\
\hline HIP 41184 & HD 70516 & 1.70 & - & 21.0 & 790 & 0.94 & 1.02 & 0.81 & VB & To14, Ma12 \\
\hline HIP 43557 & HD 75767 & 1.19 & $10.25 \mathrm{~d}$ & - & 0.1 & 0.10 & 1.00 & 0.30 & $\mathrm{SB}+\mathrm{EB}$ & SB9 \\
\hline HIP 49728 & HD 88084 & 1.30 & - & 2.3 & $79.3^{*}$ & - & 1.00 & 0.47 & $\mathrm{VB}+\mathrm{AB}$ & To14 \\
\hline HIP 78217 & HD 144061 & 1.15 & - & 2.30 & $68.1^{*}$ & - & 0.95 & 0.48 & VB & To14 \\
\hline HIP 89912 & HD 168874 & 1.42 & - & 1.50 & $42.8^{*}$ & - & 1.05 & 0.52 & $\mathrm{SB}+\mathrm{AA}+\mathrm{VB}$ & To14 \\
\hline HIP 96402 & HD 184768 & 1.28 & - & 3.20 & $123.5^{*}$ & - & 0.96 & 0.39 & VB & To14 \\
\hline HIP 104075 & HD 200746 & 1.32 & $91.91 \mathrm{yr}$ & 0.48 & 21.2 & 0.47 & 1.09 & 0.64 & VB & Ma12, To14 \\
\hline HIP 109110 & HD 209779 & 1.41 & $13.1 \mathrm{yr}$ & 0.082 & 2.9 & - & 1.04 & 0.62 & $\mathrm{SB}+\mathrm{AA}+\mathrm{VB}$ & To14 \\
\hline \multirow[t]{2}{*}{ HIP 112504} & HD 215696 & 1.37 & - & 2.71 & $91.8^{*}$ & - & 1.02 & 0.75 & VB & HIP, To14 \\
\hline & & & $420 \mathrm{~d}$ & - & - & - & 1.02 & - & SB & To14 \\
\hline HIP 115715 & HD 220821 & 1.22 & - & 0.41 & $15.0^{*}$ & - & 0.88 & 0.65 & $\mathrm{SB}+\mathrm{VB}$ & WDS, To14, No04 \\
\hline
\end{tabular}

Notes. SB: spectroscopic binaries, AB: astrometric binaries (with orbital solution), AA: stars with astrometric acceleration, VB: visual binaries, EB: eclipsing binaries, Ba: barium stars (abundance anomalies indicating a WD companion). In the semimajor axis column the asterisks indicate the projected separation in AU as measured from imaging observation. HIP 112504 is a triple system. The moderately wide visual companion and the close spectroscopic companion are listed as separated entries.

References. Cr13: Crepp et al. (2013); Ma14: Matthews et al. (2014); Sc12: Scarfe \& Griffin (2012); WDS: Mason et al. (2001); SB9: Pourbaix et al. (2004); HIP: Perryman et al. (1997); Go07: Goldin \& Makarov (2007); To14: Tokovinin (2014); To15: Tokovinin et al. (2015); Gu05: Guenther et al. (2005); Cr12: Crepp et al. (2012) Ma12: Malkov et al. (2012); No04: Nordström et al. (2004).

identified, from the extreme case of the barium-rich, ultra-fast rotator 2REJ0357+283 (Jeffries \& Smalley 1996), to the intermediate case of HD 8049 (Zurlo et al. 2013), to the planet-host GJ 86, whose slightly enhanced activity was identified through the very old kinematic age and abundance pattern (Desidera \& Barbieri 2007). This variety is the natural product of intrinsic differences in the amounts of accreted material and its angular momentum and of following evolution of the fast-rotating star, driven by magnetized stellar wind in a similar way to fastrotating young stars (Barnes 2003). As a result of the accretion and spin-up, differential rotation between the core and the envelope may develop in solar-type stars and induce mixing and Be depletion, similarly to the single slow-rotating scenario invoked by Takeda et al. (2011) and in the pre-main sequence fast rotators considered by Viallet \& Baraffe (2012). The fastest rotation occurs for stars that have recently interacted with the WD progenitor. All our program stars are characterized by slow rotation. Therefore, we expect that the interaction occurred $>1 \mathrm{Gyr}$ ago and the WD companions should have cooling ages longer than this value. The rotational mixing scenario is favoured by the recent discovery of another solar analogue with $s$-processenhancements and Be depletion (by more than a factor of fifteen, i.e., milder than in the stars considered here) but showing a rotational velocity significantly higher than solar (Schirbel et al. 2015). This star probably represents a progenitor of the stars investigated here.

\section{Concluding remarks}

Our study showed that all the four stars that are ultra-depleted in Be identified by Takeda et al. (2011) are members of moderately close binaries. Several other Be-normal stars are binaries, in some case with similar separation, indicating that binarity itself is not the decisive factor causing the Be depletion. Instead, in two cases, the visual binary HIP 64050 and the newly discovered barium star HIP 75676, the companion is a confirmed WD and, while available data are not conclusive with respect to a main-sequence companion, a WD companion is also fully compatible for the other two cases (HIP 17336 and HIP 32673). We speculate that the interaction with the WD progenitor caused alteration in the abundance pattern of the star that resulted in the severe Be depletion. We discussed possible mechanisms such as thermohaline mixing, episodic accretion, and rotational mixing.

To validate our scenario, a more detailed characterization of companions of stars with depleted or normal Be abundances should be carried out to determine their nature as WD or 
main-sequence stars. The determination of the orbital parameters of the pairs including WD companion will also be highly valuable, allowing us to constrain the original binary configuration and the amount of mass exchanges that occurred during the AGB phase.

A complementary path is to study the Be abundance of stars that are known to have a WD companion close enough to have produced significant mass and angular momentum accretion onto the companion. Main-sequence barium stars are a natural example, as mass transfer events should have been occurred while the companion (now a WD) was on the AGB, and produced carbon and s-process elements. If our scenario is correct, we expect that all barium stars are depleted in beryllium.

Acknowledgements. We thank the Haute-Provence Observatory for maintaining ELODIE and SOPHIE archives. This research has made use of the Keck Observatory Archive (KOA), which is operated by the W. M. Keck Observatory and the NASA Exoplanet Science Institute (NExScI), under contract with the National Aeronautics and Space Administration. This research has made use of the SIMBAD database, operated at CDS, Strasbourg, France and of the Washington Double Star Catalog maintained at the U.S. Naval Observatory. M.L. is a Momentum (Lendület-2014' Programme) project leader of the Hungarian Academy of Sciences.

\section{References}

Baraffe, I., \& Chabrier, G. 2010, A\&A, 521, A44

Barnes, S. A. 2003, ApJ, 586, 464

Boesgaard, A. M., \& King, J. R. 2002, ApJ, 565, 587

Boesgaard, A. M., McGrath, E. J., Lambert, D. L., \& Cunha, K. 2004, ApJ, 606, 306

Bouvier, J. 2008, A\&A, 489, L53

Busso, M., Gallino, R., \& Wasserburg, G. J. 1999, ARA\&A, 37, 239

Castelli, F., \& Kurucz, R. L. 2003, in Modelling of Stellar Atmospheres, eds.

N. Piskunov, W. W. Weiss, \& D. F. Gray, IAU Symp., 210, 20

Crepp, J. R., Johnson, J. A., Howard, A. W., et al. 2012, ApJ, 761, 39

Crepp, J. R., Johnson, J. A., Howard, A. W., et al. 2013, ApJ, 774, 1

Desidera, S., \& Barbieri, M. 2007, A\&A, 462, 345

D’Orazi, V., Magrini, L., Randich, S., et al. 2009, ApJ, 693, L31

D’Orazi, V., Biazzo, K., \& Randich, S. 2011, A\&A, 526, A103
D’Orazi, V., Biazzo, K., Desidera, S., et al. 2012, MNRAS, 423, 2789

Goldin, A., \& Makarov, V. V. 2007, ApJS, 173, 137

Grevesse, N., \& Sauval, A. J. 1998, Space Sci. Rev., 85, 161

Guenther, E. W., Paulson, D. B., Cochran, W. D., et al. 2005, A\&A, 442, 1031

Iben, Jr., I., \& Renzini, A. 1983, ARA\&A, 21, 271

Jeffries, R. D., \& Smalley, B. 1996, A\&A, 315, L19

Jeffries, R. D., \& Stevens, I. R. 1996, MNRAS, 279, 180

Jorissen, A., Van Eck, S., Mayor, M., \& Udry, S. 1998, A\&A, 332, 877

Karakas, A. I., \& Lattanzio, J. C. 2014, PASA, 31, 30

Malkov, O. Y., Tamazian, V. S., Docobo, J. A., \& Chulkov, D. A. 2012, A\&A, 546, A69

Mason, B. D., Wycoff, G. L., Hartkopf, W. I., Douglass, G. G., \& Worley, C. E. 2001, AJ, 122, 3466

Matthews, C. T., Crepp, J. R., Skemer, A., et al. 2014, ApJ, 783, L25

Mazeh, T., Prato, L., Simon, M., et al. 2002, ApJ, 564, 1007

Metchev, S. A., \& Hillenbrand, L. A. 2009, ApJS, 181, 62

Nordström, B., Mayor, M., Andersen, J., et al. 2004, A\&A, 418, 989

Pasquini, L., Bonifacio, P., Randich, S., et al. 2007, A\&A, 464, 601

Perryman, M. A. C., Lindegren, L., Kovalevsky, J., et al. 1997, A\&A, 323, L49

Pourbaix, D., Tokovinin, A. A., Batten, A. H., et al. 2004, A\&A, 424, 727

Ramírez, I., Meléndez, J., \& Asplund, M. 2009, A\&A, 508, L17

Riddle, R. L., Tokovinin, A., Mason, B. D., et al. 2015, ApJ, 799, 4

Santos, N. C., Israelian, G., García López, R. J., et al. 2004, A\&A, 427, 1085

Scarfe, C. D., \& Griffin, R. F. 2012, Rev. Mex. Astron. Astrofis., 48, 257

Schirbel, L., Meléndez, J., Karakas, A. I., et al. 2015, A\&A, 584, A116

Smiljanic, R., Pasquini, L., Bonifacio, P., et al. 2009, A\&A, 499, 103

Smiljanic, R., Pasquini, L., Charbonnel, C., \& Lagarde, N. 2010, A\&A, 510, A50

Smiljanic, R., Randich, S., \& Pasquini, L. 2011, A\&A, 535, A75

Sneden, C. A. 1973, Ph.D. Thesis, The university of Texas at Austin, USA

Stancliffe, R. J., \& Glebbeek, E. 2008, MNRAS, 389, 1828

Takeda, Y., \& Tajitsu, A. 2014, PASJ, 66, 91

Takeda, Y., Kawanomoto, S., Honda, S., Ando, H., \& Sakurai, T. 2007, A\&A, 468,663

Takeda, Y., Tajitsu, A., Honda, S., et al. 2011, PASJ, 63, 697

Tokovinin, A. 2014, AJ, 147, 86

Tokovinin, A., Mason, B. D., Hartkopf, W. I., Mendez, R. A., \& Horch, E. P. 2015, AJ, 150, 50

Viallet, M., \& Baraffe, I. 2012, A\&A, 546, A113

Vogt, S. S., Allen, S. L., Bigelow, B. C., et al. 1994, in SPIE Conf. Ser. 2198, eds. D. L. Crawford, \& E. R. Craine, 362

Zurlo, A., Vigan, A., Hagelberg, J., et al. 2013, A\&A, 554, A21 